\title{
Improving Treatment Outcomes for Tuberculosis
}

\section{Zwolska Z*}

Department of Microbiology, National Tuberculosis and Lung Diseases Research Institute, Warsaw, Poland

\begin{abstract}
Major issues are currently associated with Tuberculosis (TB) treatment, particularly in patients infected by Multidrug Resistant Tuberculosis/Extensively Drug Resistant Tuberculosis (mdr-TB/XDR-TB) resistant mycobacteria. A new threat recently reported in various Asian countries is totally Drug Resistant Tuberculosis (TDR). The presence of such Mycobacterium tuberculosis strains is disturbing also for the reasons they spread beyond the continent of Asia.

The currently recommended tuberculosis treatment regimen is not well received by patients due to its minimum six-month, complexity, and common adverse events. The prevalence of MDR-TB and XDR-TB are inversely correlated with the quality of TB control and the proper use of second-line anti-TB drugs. Moreover, cost is extraordinary high. Since the mid-1960s only two new anti-TB drugs, bedaquiline and delamandine, have come to market; however, these drugs are not available in many regions and are limited to severely resistant cases. Currently, new derivatives such as spectinoamide are of interest in tuberculosis treatment. In vitro results and animal studies are used to aid in drug development.

There is an urgent need for treatment improvement through enhancement of existing agents. Namely, individual differences in absorption and excretion of the primary anti-TB drugs, isoniazid and rifampin, require consideration. Recently, several studies attempted to evaluate the effect of anti-TB drug concentrations on treatment outcomes. Authors showed that $50-76 \%$ of the tested patients had low concentrations of INH (Isoniazid) and RMP (Rifampin). Because Therapeutic Drug Monitoring (TMD) was performed in small numbers of selected patients with comorbidities or slow treatment responses, the studies did not clearly demonstrate the effect of low drug levels on treatment outcomes. Future coordinated research is required.

New molecular tests allow for research using supervised, individualized treatment of tuberculosis. In addition, effective tuberculosis outcomes require coordinated action multiple parameters for patient detection through implementation of rapid microbiological and clinical tests as well as reliable drug resistant tests of Mycobacterium tuberculosis. This leads to a break in the chain of transmission, and prevents the spread of disease in community. Education plays in important role for patients and families concerning the causes of disease and prevention methods. Additionally, medical staff should also themselves improve the level of diseases knowledge. Behaviour changes in tuberculosis infection control among medical personnel is also required. Keep in mind that one of the reasons for the relapse of tuberculosis is its disregard.
\end{abstract}

Keywords: Tuberculosis treatment outcomes; Treatment cost; Pharmacokinetic variability; Individualized therapy; Education

\section{Introduction}

\section{Epidemiology of tuberculosis}

Although TB treatment has existed for more than 70 years, it is considered a failure of modern therapy. Despite a known etiology and drugs available over the last 135 years, tuberculosis is one of the oldest infectious diseases and has evolved into a new stronger enemy, drugresistant tuberculosis. In 2016, an estimated 580,000 people suffered from drug-resistant TB worldwide. Drug resistance rates are one of the most important aspects in the TB control program as well as in drug-resistant TB, especially in Extensively Drug-resistant (XDR) TB. Inadequate and incomplete treatment including poor treatment adherence, has led to a newer form of drug resistance. Recent studies have described XDRTB strains from all continents. Worldwide prevalence of XDR-TB is estimated to be $6.6 \%$ in all the examined countries among multi-drug resistant M. tuberculosis strains, $6.5 \%$ in industrialized countries, $13.6 \%$ in Russia and Eastern Europe, $1.5 \%$ in Asia, $0.6 \%$ in Africa and the Middle East [1].

Since 2006, at least 100 countries have reported cases of XDR TB. While tuberculosis is curable in almost all patients, MDR-TB is difficult to treat with a cure rate of $50-60 \%$. The isolation of Totally Drug Resistant (TDR-TB) strains from MDR-TB patients' strains is disturbing also for the reasons they spread beyond the continent of Asia. Results of treatment of tuberculosis TDR so far are unknown. The pressing question is now how to control and treat such cases [2].

The currently recommended treatment regimen for drug-resistant tuberculosis is difficult to bear by patients due to adverse effects and by the government because of high health care costs. A recent survey estimates the cost of drugs to treat one patient in Europe with MDRTB is 23,000 Euro. For a XDR-TB patient, the cost rises to 93,000 Euro $[3,4]$. Such financial resources are not available for majority of patients affected by MDR and XDR-TB particularly in low- or middle-income countries and countries with military conflicts. The emergence of XDR-TB has been reported worldwide. According to the World Health Organization (WHO), the numbers of notified cases with MDR/XDR TB increased in the Ukraine before the war from 3482, 5336, 4306 and

*Corresponding author: Zwolska Z, Department of Microbiology, National Tuberculosis and Lung Diseases Research Institute, 26 Płocka, 01-138 Warsaw, Poland, Tel: 48-22-43-12-182; Fax: 48-2243-12-182; E-mail: z.zwolska@igichp.edu.pl

Received May 22, 2017; Accepted June 12, 2017; Published June 22, 2017

Citation: Zwolska Z (2017) Improving Treatment Outcomes for Tuberculosis. J Bioequiv Availab 9: 442-446. doi: 10.4172/jbb.1000341

Copyright: (c) 2017 Zwolska Z. This is an open-access article distributed under the terms of the Creative Commons Attribution License, which permits unrestricted use, distribution, and reproduction in any medium, provided the original author and source are credited. 
6934 up to 10,585 between 2009 and 2013. The European Centers for Disease Prevention and Control reported 3522 patients with MDR/XDR living in the Ukraine in 2012, compared to 1421 in all European Union/ European Economic Area countries combined [5].

The WHO estimated 9.6 million new global TB cases in 2016: 5.4 million males, 3.2 million females, and 1.0 million children. Almost $80 \%$ of active $\mathrm{TB}$ cases and $95 \%$ of $\mathrm{TB}$ deaths occur in low- or middleincome countries [6]. The disease still poses a serious public health problem worldwide and remains the leading cause of death due to a single infectious agent $[7,8]$. Despite that epidemiology of TB in Poland since 1945 has been constantly improving, in 2015 the incidence of TB in Poland was higher than the mean incidence in the European Union and European Economic Area (17.4 v 12.7 per 100,000). Tuberculosis remains the most common single cause of death due to an infectious agent in Poland $[9,10]$.

\section{Treatment of Tuberculosis}

Early diagnosis and effective treatment of TB and are key elements in reducing infection transmission. The current first-line treatment for $\mathrm{TB}$ is a multidrug regimen that consists of isoniazid, rifampicin, pyrazinamid, and ethambutol (HRTZE). This drug course must be maintained for at least six months to achieve a high cure rate. The WHO has set an international target value for favourable treatment outcomes at $85 \%$ published recommendations for assessing outcomes of tuberculosis treatment in the 1990 's, with a revised version published in 1998 [11,12]. However, in many highly developed countries with good treatment facilities, outcomes have still not reached the targets set by the WHO. Poland belongs to the group where result did not meet the WHO requirements. In 2015, the success rate of the pulmonary TB treatment in Poland was $57 \%$ and Extrapulmonary Tuberculosis (ExtraTB) treatment was $57.6 \%$ [9].

Treatment of MDR-TB is difficult as current regimens, when compared to those used to treat drug-susceptible TB, are less effective but more costly, toxic, and lengthy Due to the expanded use of secondline drugs (aminoglycosides, fluoroquinolones, cycloserine, etionamide, and others) in patients with MDR TB, the prevalence of XDR TB is increasing.

In 2000, the Green Light Committee (GLC) was formed within the Stop TB Partnership and WHO in order to increase access to highquality, second-line anti-TB drugs at low prices, to prevent additional drug resistance, and to contribute evidence toward policy development. By 2011, 255 project applications to the GLC had been approved, covering more than 130,000 patients with MDR TB $[13,14]$.

The currently available TB treatment regimen presents a number of weak points: duration and complexity of therapy, decreased adherence to treatment, common adverse events in response to anti-TB drugs, and increasing incidence of MDR and XDR tuberculosis [15]. There is an urgent need to improve treatment by either enhancing the applications of existing agents or introducing new drugs. Only two new anti-TB drugs, bedaquiline and delamandine, have come to the market since the mid-1960s, and the use of these medicines is currently limited to the most severely resistant cases. However, these drugs are not available in many countries. Current antibiotic-based drug resistant TB treatment strategies are arduous for patient causing many adverse effects including hearing impairment and psychosis, as well as some practical problems for families, communities, health systems, and livelihoods.

\section{New drugs}

An ideal drug is expected to reduce treatment duration, show acceptable tolerability, act against MDR/XDR tuberculosis, demonstrate minimal interactions with antiretroviral drug in HIV/AIDS patients, and act against latent infection. However, it is still an open question on how to develop a perfect drug? Many coordinated actions are needed to increase the interest of scientists and sponsors to search for new anti-TB drugs. The rapid search for new drugs for tuberculosis is associated with a number of obstacles to overcome [16]. The first obstacle is the cost of developing a new drug that has been estimated at 115-240 million dollars. Thus, from an economical point of view, it does not appear to be profitable, because the cost of a standard regimen is only about 11 dollars per patient [17]. The next challenge is identification of new, effective compounds showing bactericidal and sterilizing activity against Mycobacterium tuberculosis however, no reliable animal model for the preclinical study has been developed and clinical testing of new anti-TB drugs would be time-consuming. Therefore, urgent attention should be paid to the development of new drugs, requiring greater involvement of large pharmaceutical industries. Each year, scientific reports discuss a series of new chemical compounds that exhibit anti-TB activity in vitro that could be promising in clinical trials. One group of distinct antibiotics is a new semi synthetic series of spectinamide analogs with selective ribosomal inhibition and excellent narrow-spectrum antitubercular activity, which is associated with pump-mediated efflux. Spectinomycin is chemically similar to aminoglycoside and has previously been used to treat gonorrheal infections. Unlike aminoglycosides, spectinomycin has a high safety margin, and exhibits minor side effects with no nephro- or ototoxtoxicity when administered for a short term at high therapeutic doses. In multiple murine infection models, spectinomycin was well tolerated, significantly reduced lung mycobacterial burden, and increased survival. In vitro studies demonstrated a lack of cross-resistance with existing anti-TB drugs and an excellent pharmacological profile $[18,19]$. Microbial and pharmacological testing of the spectinamides as a novel antitubercular agents has demonstrated properties desired for preclinical candidacy.

\section{Therapeutic Drug Monitoring}

Tuberculosis requires treatment using three to five different drugs simultaneously depending on the patient category. In most tuberculosis patients, drug resistance predominantly arises as a result of multiple interruptions of treatment continuity. When using single-drug formulations, patients are more prone to interrupt their treatment on some drugs while not on others, thereby creating a risk of monotherapy and selection of drug-resistant mutants. Anti-tuberculosis drugs can be given as single-drug formulations or as fixed-dose combination formulations (FDCs) where two or more anti-tuberculosis drugs are present in fixed proportions in the same formulation. The WHO, the International Union Against Tuberculosis and Lung Disease (IUATLD) The American Thoracic Society (ATS), Centers for Disease Control and Prevention (CDC-P), and the Infectious Diseases Society of America (IDSA) all advocate the replacement of single-drug preparations with FDC (fixed dose combination) tablets as the primary treatment for tuberculosis [20]. Fixed dose combination tablets provide a simple approach to deliver multiple drugs at the correct dosage as all the necessary active substances are combined in a single tablet. In Poland, the majority of patients (95\%) receive combination tablets isoniazid plus rifampin at the beginning of therapy.

Therapeutic drug monitoring is a standard clinical technique used for many infectious diseases. This technique is helpful in indicating the necessary time of treatment and avoidance of side effects [21]. However, to assess drug quality, bioavailability and bioequivalence should be monitored [22]. 
Standard TB treatment does not take into account individual patient characteristics such as ethnicity, gender, age, and unique genetic properties. All of these factors can affect drug bioavailability by modifying their absorption and excretion profile. Therefore, the same doses of a given drug may show unequal effectiveness in different patients. Without this knowledge part of the patients may not be cured or the treatment takes longer period of time than the standard treatment duration (Figure 1).

Among all the anti-TB drugs RMP and INH take the most important position in hierarchy. They have broad spectrum activity, good bioavailability, moderate side effects, excellent penetration into the phagocytes, and low cost making them ideal anti-TB drugs. However, these drugs have a relatively high frequency of spontaneously developed resistance in $M$. tuberculosis, precluding their use in monotherapy. RMP and INH, when used in combination with other drugs, did not prevent resistance of mycobacteria [1].

Blood serum concentrations of rifampin and isoniazid are not always proportional to the dose administered [23,24]. As for RMP, it is desacetylated in the liver and large variability in the absorption rate and serum concentration values have been observed. In the 1980's, in many countries including Poland, the above-mentioned phenomenon was studied in detail in an attempt to find the explanation [24,25].

In clinical and in vitro studies found some regularity that affect the concentration of the RMP in serum. Gender and age of patients as well as the competitive impact of food were the most important factors affecting the absorption of the drug [26,27].

In Polish experiments, the serum levels of RMP were analysed in relation to sex, age, and body weight. It was revealed that, in women, the drug concentration was approximately twice as high as in men. The level of RMP in women up to 40 years of age were higher than in older women. In men, no differences as related to age were found. Concerning the impact of food on RMP bioavailability, patients who received the drug after fasting had serum levels 30 to $100 \%$ higher than those who received the drug after breakfast. The differences were found to be statistically significant.
In addition, the impact of food on INH absorption has been examined. The results show that $\mathrm{C}_{\max }, \mathrm{t}_{\max }$, and AUC in both fast and slow acetylators are significantly decreased after ingestion of INH with breakfast [28]. Because of this, Polish patients are recommended to take RMP and INH prior to consuming breakfast.

In theory, food intake exerts a complex influence on drug bioavailability. It may interfere with tablet disintegration, drug dissolution, and drug transit through the gastrointestinal tract. Additionally, food may also affect the metabolic transformation of drugs in the gastrointestinal wall and the liver. Therefore, understanding the influence of food is critical in TB treatment where the drugs are taken on a more infrequent basis. Despite this, patients are not informed about the necessity of taking RMP prior to meals.

The pharmacogenetic determinants of rifampin exposure are poorly understood. Recently, Chigutsa et al. [29] have observed worse treatment outcomes in African patients and discovered RMP plasma concentration variability in TB patients, which was explained by differences in HIV infection status, sex, formulation, and weight. The authors suggested that the variability observed in patient's drug concentrations may partially result from differences in genes encoding drug-metabolizing enzymes or transporters (coded by ABCBI and SLCOIBI genes). SLCOIBI rs4149032 was present in most patients and was associated with substantially reduced rifampin exposure. The data suggests that the standard recommended dose of RMP for all patients should be reconsidered for South Africans. The phenomenon of INH acetylation is known for a long time and had been tested intensively from the first years of INH using in treatment of tuberculosis, in practical as well cognitive aspects. INH is metabolized in the liver by an acetylation process in a polymorphic profile, and two clearly distinct types, Fast (FastAC) and Slow Acetylators (SlowAC) reach different serum concentrations even after the administration of the same drug dose. In addition, the process of elimination is much longer in the SlowAC than in the FastAC. Figure 2 shows differences in the bioavailability of INH in serum fast and slow acetylators. Isoniazid bioavailability following administration of similar doses per kg body weight in healthy volunteers for FastAC and SlowAC showed statistically significant

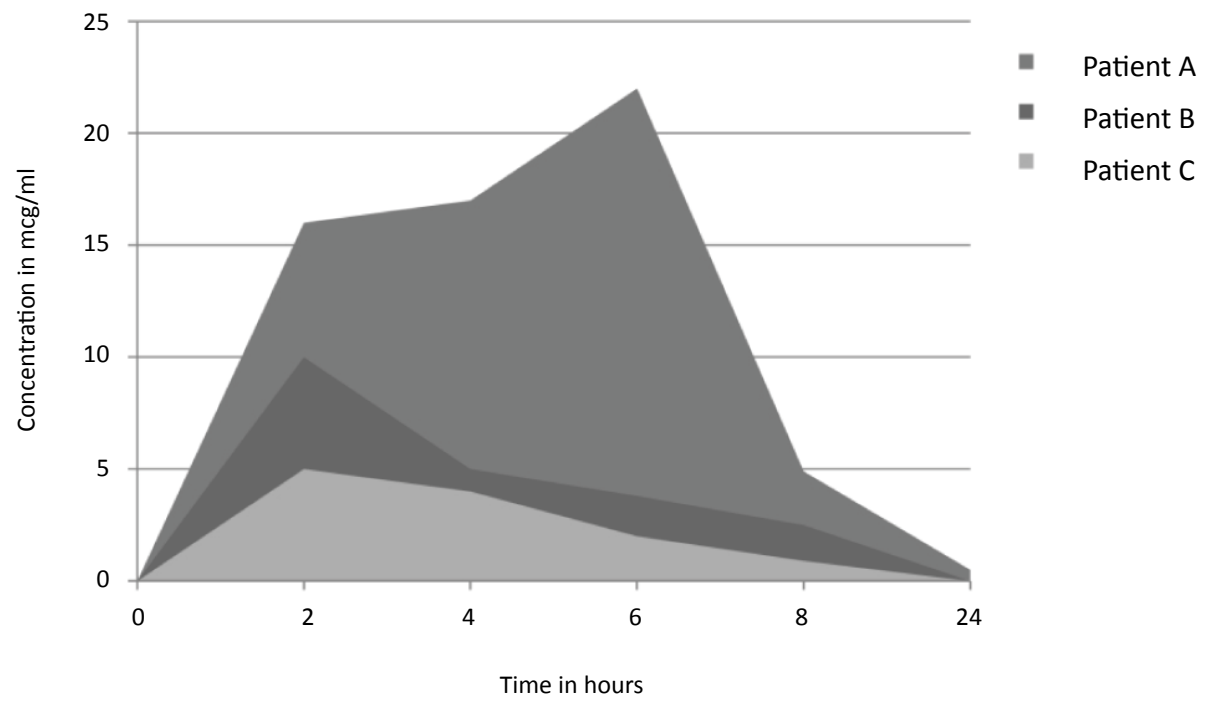

Figure 1: Different types of RMP absorption and excretion in TB Polish patients. 


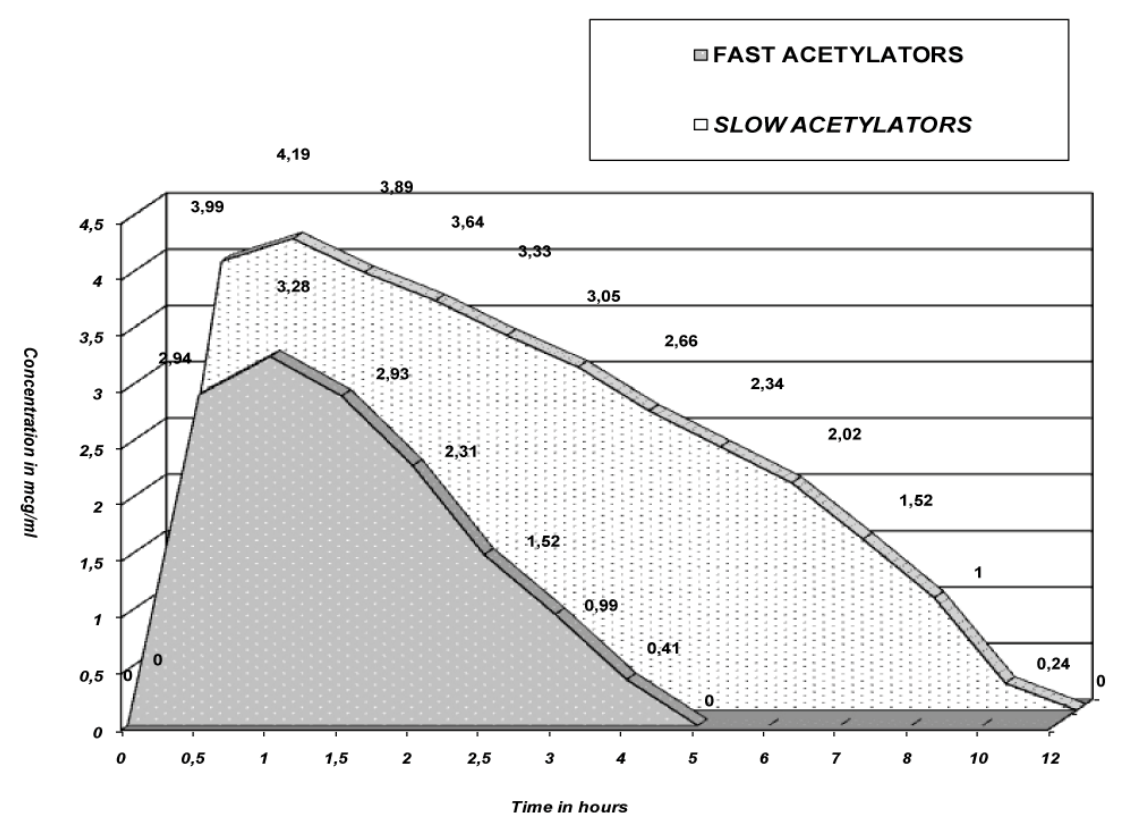

Figure 2: Mean plasma concentration of isoniazid in fast and slow acetylator's Polish healthy volunteers.

differences. Bioavailability of INH expressed by $\mathrm{C}_{\text {max }}$ and AUC was much lower in FastAC than in SlowAC

Serum bactericidal INH concentration in some FastAC is detectable no longer than 2.5-3 $\mathrm{h}$ up after administration of the drug. Keeping in mind that INH is administered to the patients once a day, it is important to know if the drug concentration is sufficient for the desired outcome.

Efficacy and safety of administered INH in tuberculosis may be hampered (as demonstrated by in vitro testing) by easy transformation of susceptible mycobacteria into resistant population, a phenomenon that can occur with suboptimal doses. So, it is suspected that a polymorphic type of acetylation in humans may have an impact on the treatment effects of INH $[30,31]$. The main question that arises is whether the fast acetylators have more exposure to INH resistance than slow acetylators. In a study by Parkin et al. differences in the disposition kinetics of isoniazid have been observed between SlowAC and FastAC. Slow acetylators had four- to six-fold higher serum isoniazid concentrations at 2-6 $\mathrm{h}$ following oral administration than rapid acetylators, prompting the authors to propose individualized isoniazid dosing regimen [32]. In 1973, a WHO report discussed the importance of determining phenotype of acetylation during TB therapy [33].

\section{Summary}

In summary, the supervision and control of tuberculosis requires more than just discovering new drugs. The fight against tuberculosis is not only a medical activity but also concerns social and economic issues. The diagnostic and therapeutic facilities of health care should be improved. There is an urgent need to develop more effective treatment regimens against MDR/XDR TB. Individual differences in drug absorption and excretion should be taken into account. Medical staff should also themselves improve the level of diseases knowledge. Behavior changes in tuberculosis infection control among medical personnel is also required. Transmission of Mycobacterium tuberculosis from individuals with TB to other patients and to Health Care Workers (HCWs) is a well-known nosocomial hazard $[34,35]$.
In order to prevent drug resistance, plasma concentration testing of at least two major drugs RMP and INH should be monitored. Proper control of TB requires improvement on disease detection, transmission prevention, and prevention of drug resistance. Finally, it should be noted that, despite of extensive knowledge of monitoring treatment, current global, epidemiological situation of $\mathrm{TB}$ is not conducive for individualizing dosage according to the different types of drug absorption.

A brief overview of the current issues associated with escalating and dangerous issues of drug-resistant TB does not cover all aspects. Medical services, as well as financiers and managers, are needed to prevent deterioration of the current situation.

At the end, it is worth to quote the letter of Executive Director IUATLD doctor Jose Luis Castro to doctor Margaret Chan WHO Director of 1 March 2017. (Union letter to WHO protests unjustified of TB antibiotics global priority list).

"We are writing to you in strong protest of the fact that tuberculosis was left off- in fact, was not considered for inclusion within- WHO's first-ever Global priority list of antibiotic-resistant bacteria to guide research, discovery, and development of new antibiotics, published 27 February 2017."

\section{References}

1. Günther G, Gomez GB, Lange C, Rupert S, van Leth F, et al. (2015) Availability, price and affordability of anti-tuberculosis drugs in Europe: a TBNET survey. Eur Respir J 45: 1081-1088.

2. Udwadia ZF, Amale RA, Ajbani KK, Rodrigues C (2012) Totally drug-resistant tuberculosis in India. Clin Infect Dis 54: 579-581.

3. Diel R, Vandeputte J, de Vries G, Stillo J, Wanlin M, et al. (2014) Costs of tuberculosis disease in the European Union: a systematic analysis and cost calculation. Eur Respir J 43: 554-565.

4. Tanimura T, Jaramillo E, Weil D, Raviglione M, Lonnroth K (2014) Financial burden for tuberculosis patients in low- and middle-income countries: a systematic review. Eur Respir J 43: 1763-1775. 
5. Dudnyk A, Rzhepishevska O, Kutsyna G, Lange K (2015) Multidrug-resistant tuberculosis in Ukraine at the time of military conflict. Int J Tuberc Lung Dis 19: 492-496.

6. European Center for Disease Prevention and Control/WHO Regional Office for Europe (2014) Tuberculosis surveillance and monitoring in Europe. Stockholm, Sweden.

7. World Health Organization (2015) Global Tuberculosis report. WHO/HTM/ TB/2015.22.Geneva, Switzerland.

8. European Centre for Disease Prevention and Control/WHO regional Office for Europe (2016) Tuberculosis surveillance and monitoring in Europe Stockholm, Sweden.

9. Tuberculosis and Lung Diseases in Poland (2015) Ed: Maria KorzeniewskaKosela National Tuberculosis and Lung Diseases Research Institute Department of Epidemiology, Warsaw.

10. Bryta M, Dziankowska-Zaborszczyk E, Maniecka-Bryta I (2017) Standard expected years of life lost due to tuberculosis in Poland. Int J Tuberc Lung Dis 21: 202-207.

11. WHO Tuberculosis Programme (1994) WHO Tuberculosis Programme: framework for effective tuberculosis control. World Health Organization, Geneva.

12. Veen J, Raviglione M, Rieder HL, Migliori GB, Graf P, et al. (1998) Standardized tuberculosis treatment outcome monitoring in Europe. Recommendations of a Working group of the World Health Organization (WHO) and the European Region of the International Union against Tuberculosis and Lung Disease (IUATLD) for uniform reporting by cohort analysis of treatment outcome in tuberculosis patients. Eur Respir J 12: 505-510.

13. Dalton T, Cegielski P, Akksilp S, Asencios L, Caoli JC, et al. (2012) prevalence of risk factors for resistance to second-line drugs in people with multidrugresistant tuberculosis in eight countries: a prospective cohort study. The Lancet 380: 1406-1417.

14. World Health Organization (2010) Multidrug and extensively drug-resistant TB (M/XDR-TB): 2010 global report on surveillance and response.

15. van den Boogaard J, Gibson SK, Kisanga ER, Boeree JB, Aarnoutse RE (2009) New drug against tuberculosis: problems, progress, and evaluation of agents in clinical development. Antimicrob Agents Chemother 53: 849.

16. Gardner CA, Acharya T, Pablos-Méndez A (2005) The global alliance for tuberculosis drug development-accomplishments and future directions. Clin Chest Med 26: 341-347.

17. O'Brien RJ, Nunn PP (2001) The need for new drugs against tuberculosis. Obstacles, opportunities, and next steps. Am J Respir Crit Care Med 163: 1055-1058.

18. Novak E, Schlagel CA, LeZotte LA, Pfeifer RT (1974) The tolerance of high dose intravenous spectinomycin therapy in man. J Clin Pharmacol 14: 442-447.

19. Lee RE, Hurdle JG, Liu J, Bruhn DF, Matt T, et al. (2014) Spectinamides: a new class of semisynthetic antituberculosis agents that overcome native drug efflux. Nat Med 20: 152-158.
20. Blomberg B, Spinaci S, Fourie B, Laing R (2001) The rationale for recommending fixed-dose combination tablets for treatment of tuberculosis. Bull World Health Organ 79: 61-68.

21. Peloquin CA (2002) Therapeutic drug monitoring in the treatment of tuberculosis. Drugs 62: 2169-2183.

22. Zwolska Z, Niemirowska-Mikulska H, Augustynowicz-Kopec E, Walkiewicz $\mathrm{R}$, Stambrowska $\mathrm{H}$, et al. (1998) Bioavailability of rifampicin, isoniazid and pyrazinamide from fixed- dose combination capsules. Int J Tuberc Lung Dis 2: 824-830.

23. Pasipanodia J, Srivastava S, Gumbot T (2012) Methaanalysis of clinical studies supports the pharmacokinetic variability hypothesis for acquired drug resistance and failure of antituberculosis therapy. Clin Infect Dis 55: 169-177.

24. Golka K, Selinski S (2016) NAT2 Genotype and Isoniazid Medication in Children. EBioMedicine 11: 11-12.

25. Acocella G (1983) Pharmacokinetics and metabolism of rifampicin in humans Rev Inf Dis 5: 428-431.

26. Zwolska-Kwiek Z (1974) Variability in rifampicin concentrations in the serum of patients with tuberculosis. Tuberculosis et Pneumonologia XLII: 165-173.

27. Zwolska-Kwiek Z, Lawicka E, Wasowska H (1975) Comparison of rifampicin serum levels after the administration before breakfast and 3 hours after breakfast. Tuberculosis et Pneumonologia 43: 151-156

28. Zwolska Z, Niemirowska-Mikulska H, Augustynowicz-Kopec E (1997) [Influence of food on the bioavailability of isoniazid in health volunteers. Pneumonol Alergol Pol 66: 412-421.

29. Chigutsa E, Visser ME, Swart E, Denti P, Pushpakom S, et al. (2011) The SLOCO1B1 rs419032 polymorphism is highly prevalent in South Africans and is associated with reduced rifampin concentrations: dosing implications. Antimicrob Agents Chemother 55: 4122-4127.

30. Park JS, Lee JY, Lee YJ, Kim SJ, Cho YJ, et al. (2016) Serum levels of antituberculosis drugs and their effect on tuberculosis treatment outcome. Antimicrob Agents Chemother 60: 92-98.

31. Jung JA, Kim TE, Lee $H$, Jeong BH, Park HY, et al. (2015) A proposal for an individualized pharmacogenetic-guided isoniazid dosage regimen for patients with tuberculosis. Drug Des Dev Ther 9: 5433-5438.

32. Parkin DP, Vandenplas S, Botha FJ, Vandenplas ML, Seifart HI, et al. (1997) Trimodality of isoniazid elimination: phenotype and genotype in patients with tuberculosis. Am J Respir Crit Care Med 155: 1717-1722.

33. WHO Scientific Group on Pharmacogenetics (1973) Pharmacogenetics: Report of WHO Scientific Group. World Health Organization, Technical report Series, Geneva.

34. Menzies D, Joshi R, Pai M (2007) Risk of tuberculosis infection and disease associated with work in health care settings. Int J Tuberc Lung Dis 11: 593-605.

35. Sotgiu G, Arbore AS, Cojocariu V, Piana A, Ferrara G, et al. (2008) High risk of tuberculosis in health care workers in Romania. Int J Tuberc Lung Dis 12 : $606-611$. 